Response to: 'Infliximab and CT-P13 immunogenicity assessment in PLANETAS and PLANETRA main and extension studies: utility of laboratory methods description' by Francesca Meacci et al

We sincerely appreciate the interest shown by Dr Francesca Meacci and colleagues in the comparative immunogenicity evaluations of reference infliximab and CT-P13 (biosimilar infliximab) that were performed in the PLANETAS and PLANETRA clinical trials and their trial extension studies. ${ }^{1-7}$ In addition, we acknowledge their specific comments regarding the potential value of the Gyrolab platform and its many practical advantages as a laboratory method. ${ }^{7}$

In order to provide robust antidrug antibody (ADA) data, both electrochemiluminescence and Gyrolab (Gyros AB, Uppsala, Sweden) were used for PLANETRA and PLANETAS studies. To ensure a thorough evaluation of an ADA response (or absence of such a response), it is usually necessary to perform the following three procedures: (1) screening, (2) confirmation and (3) characterisation. The screening assay determines overall ADA status, categorising samples as ADA positive or ADA negative according to the presence or absence of ADAs, respectively. Next, the specificity of any ADAs detected during screening should be tested in a confirmation assay (also termed a 'competitive' or 'inhibition' assay). In this step, free (ie, nonlabelled) drug is added to fluorescence-labelled samples and the magnitude of inhibition of the fluorescence signal is then monitored. ${ }^{8} 9$

Characterisation assays can be used to describe several different aspects of an ADA response, including the neutralising activity of ADA-positive samples. In PLANETAS and PLANETRA, samples testing positive for ADAs were further analysed to check the neutralising activities of the detected ADAs. Neutralising antibodies (NAbs) were detected using a state-of-the-art biotechnology system known as the Gyrolab platform. Of note, the proportion of patients who provided samples that tested positive for NAbs was similar in patients who were maintained on CT-P13 in the PLANETAS and PLANETRA extension studies ('maintenance group') and those who switched from reference infliximab to CT-P13 in these studies ('switch group'). 56

Gyrolab is a flow-through automated micro-immunoassay platform and one of a number of ligand-binding assay technology platforms that are now available. ${ }^{10}$ It has been used for measurement of NAbs in PLANETAS and PLANETRA and for quantification of rituximab concentrations in human serum. ${ }^{11}$ This miniaturised microfluidic immunoassay (which uses a compact-sized column and low reaction volume) is based on a succession of principles including capillary action, hydrophobicity, centrifugal force and protein-protein interaction. ${ }^{12}{ }^{13}$ To briefly describe the assay, biotinylated capture antibodies specific for tumour necrosis factor (TNF) plus streptavidin-coated beads and then recombinant full-length TNF proteins are loaded into the column by capillary action and centrifugal force. After this, samples preincubated with fluorescence-labelled CT-P13 or reference infliximab are passed through the column to allow binding of samples to TNF. If the samples retain NAbs, fluorescence-labelled reference infliximab or CT-P13 are unable to bind to TNF, resulting in no generation of fluorescence signals. At each step, hydrophobic barriers prevent reactants from flowing. The magnitude of the reduction in the intensity of the detected fluorescence signals reflects the amount of NAbs present in a patient's sample, when compared with a positive or negative control.

Beyond minor limitations and other considerations, the Gyrolab platform has many advantages over other methods including a rapid analysis time, high sensitivity and accuracy, good reproducibility, ease-of-use, reduced error rate and bias (by virtue of its automated processes) and nanolitre sample volumes. ${ }^{8}$ 10-13 We hope that our studies represent an enterprising attempt to understand, and expand the availability and reliability of, immunogenicity assessments during drug development. To provide further information on the topics described above, we plan to publish a separate article focused on the methodology of immunogenicity testing in the near future.

\section{Jürgen Braun}

Correspondence to Professor Jürgen Braun, Rheumazentrum Ruhrgebiet, Claudiusstr. 45, Herne 44649, Germany; j.braun@rheumazentrumruhrgebiet.de

Contributors JB contributed to the drafting of this response and revising it critically for important intellectual content, and final approval of the version to be published. Writing support was provided by Sang Wook Yoon PhD (an employee of Celltrion Healthcare Co., Ltd, Incheon, Republic of Korea). Editing support was provided by Rick Flemming PhD, CMPP (Aspire Scientific Ltd, Bollington, UK).

Funding Celltrion Healthcare Co., Ltd.

Competing interests JB has received honoraria for talks, advisory boards, paid consultancies or grants for studies from Abbvie (Abbott), Amgen, Biogen, Boehringer Ingelheim, Bristol-Myers-Squibb, Celgene, Celltrion, Centocor, Chugai, EBEWE Pharma, Epirus, Janssen, Hospira, Medac, MSD (Schering-Plough), Mundipharma, Novartis, Pfizer (Wyeth), Roche, Sanofi-Aventis and UCB.

Provenance and peer review Commissioned; internally peer reviewed.

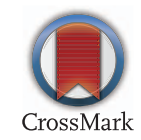

To cite Braun J. Ann Rheum Dis 2016;75:e63.

Accepted 11 July 2016

Published Online First 28 July 2016

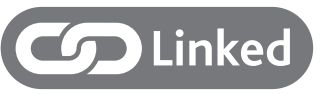

- http://dx.doi.org/10.1136/annrheumdis-2016-210078

Ann Rheum Dis 2016;75:e63. doi:10.1136/annrheumdis-2016-210091

\section{REFERENCES}

1 Park W, Hrycaj P, Jeka S, et al. A randomised, double-blind, multicentre, parallel-group, prospective study comparing the pharmacokinetics, safety, and efficacy of CT-P13 and innovator infliximab in patients with ankylosing spondylitis: the PLANETAS study. Ann Rheum Dis 2013;72:1605-12.

2 Yoo DH, Hrycaj P, Miranda P, et al. A randomised, double-blind, parallel-group study to demonstrate equivalence in efficacy and safety of CT-P13 compared with innovator infliximab when coadministered with methotrexate in patients with active rheumatoid arthritis: the PLANETRA study. Ann Rheum Dis 2013;72:1613-20.

3 Park W, Yoo DH, Jaworski J, et al. Comparable long-term efficacy, as assessed by patient-reported outcomes, safety and pharmacokinetics, of CT-P13 and reference infliximab in patients with ankylosing spondylitis: 54-week results from the randomized, parallel-group PLANETAS study. Arthritis Res Ther 2016;18:25.

4 Yoo DH, Racewicz A, Brzezicki J, et al. A phase III randomized study to evaluate the efficacy and safety of CT-P13 compared with reference infliximab in patients with active rheumatoid arthritis: 54-week results from the PLANETRA study. Arthritis Res Ther 2016;18:82.

5 Park W, Yoo DH, Miranda P, et al. Efficacy and safety of switching from reference infliximab to CT-P13 compared with maintenance of CT-P13 in ankylosing spondylitis: 102-week data from the PLANETAS extension study. Ann Rheum Dis 2016; Published Online First 26 Apr 2016. doi:10.1136/annrheumdis-2015-208783 
6 Yoo DH, Prodanovic N, Jaworski J, et al. Efficacy and safety of CT-P13 (biosimilar infliximab) in patients with rheumatoid arthritis: comparison between switching from reference infliximab to CT-P13 and continuing CT-P13 in the PLANETRA extension study. Ann Rheum Dis 2016; Published Online First: 29 Apr 2016. doi:10.1136/annrheumdis-2015-208786

7 Meacci $F$, Manfredi M, Infantino $M$, et al. Infliximab and CT-P13 immunogenicity assessment in PLANETAS and PLANETRA main and extension studies: utility of laboratory methods description. Ann Rheum Dis 2016:75:e62.

8 Mikulskis A, Yeung D, Subramanyam M, et al. Solution ELISA as a platform of choice for development of robust, drug tolerant immunogenicity assays in support of drug development. J Immunol Methods 2011;365:38-49.

9 Gunsior M. Implications of Immunogenicity in Drug Development. Global Bioanalytical Services White Paper (Covance). http://www.covance.com/content/
dam/covance/assetLibrary/whitepapers/adawhitepaperpdf.pdf (last accessed 5 Jul 2016).

10 Leary BA, Lawrence-Henderson R, Mallozzi C, et al. Bioanalytical platform comparison using a generic human IgG PK assay format. J Immunol Methods 2013;397:28-36.

11 Liu XF, Wang X, Weaver RJ, et al. Validation of a Gyrolab ${ }^{\mathrm{TM}}$ assay for quantification of rituximab in human serum. J Pharmacol Toxicol Methods 2012;65:107-14.

12 Inganäs $M$, Dérand $H$, Eckersten $A$, et al. Integrated microfluidic compact disc device with potential use in both centralized and point-of-care laboratory settings. Clin Chem 2005;51:1985-7.

13 Given AM, Whalen PM, O'Brien PJ, et al. Development and validation of an alpha fetoprotein immunoassay using Gyros technology. J Pharm Biomed Anal 2012;6465:8-15. 